\title{
Comparison of the endocytoscopic and clinico- pathologic features of colorectal neoplasms
}

Authors

Institutions
Kenichi Takeda', Shin-ei Kudo', Masashi Misawa', Yuichi Mori', Toyoki Kudo', Kenta Kodama', Kunihiko Wakamura', Hideyuki Miyachi', Eiji Hidaka', Fumio Ishida', Haruhiro Inoue ${ }^{2}$

1 Digestive Disease Center, Showa University, Yokohama Northern Hospital, Kanagawa, Japan

${ }^{2}$ Digestive Disease Center, Showa University, Koto Toyosu Hospital, Tokyo, Japan submitted:

20. July 2015

accepted after revision:

18. January 2016

\section{Bibliography}

DOI http://dx.doi.org/

10.1055/s-0042-101753

Published online: 24.3.2016

Endoscopy International Open

2016; 04: E397-E402

(c) Georg Thieme Verlag KG

Stuttgart $\cdot$ New York

E-ISSN 2196-9736

\section{Corresponding author \\ Shin-ei Kudo, MD PhD}

Digestive Disease Center Showa University

Northern Yokohama Hospital

35-1 Chigasaki-Chuo

Tsuzuki

Yokohama 224-8503

Japan

Fax: +81-45-9497535

kudos@med-showa-u.ac.jp
Background and aim: Permeation of a vein or lymphatic vessel by a tumor is a key risk factor for lymph node metastasis. We examined the features of colorectal tumor vessel permeation using endocytoscopy, an ultra-high magnifying endoscopic system combined with a narrow-band imaging capability (EC-NBI).

Patients and methods: We examined 188 colorectal lesions using EC-NBI before treatment was started. We measured the diameters of tumor vessels on EC-NBI images. We used the tumor vessel diameter (the mean diameter of four tumorassociated vessels) and the variation in tumor vessel caliber (the difference between the maximum and minimum diameters of the vessels expressed as a proportion) to judge changes in vessel formation. We examined the relationship between these variables and the extent of venous or lymphatic vessel permeation (vessel invasion)

\section{Introduction}

$\nabla$

When planning treatment for colorectal tumors, it is important to predict the risk of metastasis to the lymphatic system or other organs. Pit pattern classification, assessed using magnifying chromoendoscopy, is reportedly a useful means of judging whether a lesion is neoplastic or has malignant potential, and establishing the depth of invasion in cases of invasive carcinoma [1,2]. Observing the vascular patterns of tumors with a narrow-band imaging (NBI) system, which uses the absorbance of hemoglobin, is also reportedly useful for evaluating the potential for malignancy and depth of tumor invasion [3-7], and consequently, allows the risk of metastasis to be estimated. However, tumor venous or lymphatic vessel permeation is also risk factor for tumor metastasis; both are usually determined by histopathologic examination of resected specimens [8]. Venous and lymphatic vessel permeation (tumor vessel invasion) is usually diagnosed by immuno- established by immunohistochemical examination of the resected specimen using monoclonal antibodies against the CD34 and D2 - 40 antigens. We also analyzed the relationships between tumor vessel diameter, tumor vessel caliber variation, and depth of tumor invasion.

Results: There were significant differences in tumor vessel diameter and caliber variation between tumors in situ and T1-T3 carcinomas. In T1 carcinomas, larger tumor vessel diameter and greater tumor vessel caliber variation were significantly associated with venous permeation. In T2 and T3 carcinomas, greater tumor vessel caliber variation was significantly associated with venous permeation.

Conclusions: The vessel diameter and caliber variation of colorectal tumor microvasculature are associated with depth of invasion and venous permeation, especially in $\mathrm{T} 1$ carcinomas.

histochemical examination of the resected specimen stained with monoclonal antibodies against CD34 and D2 - 40, respectively. In cases when venous or lymphatic vessel permeation is found in endoscopically resected specimens, additional surgical lymph node resection is recommended and informs the clinical decision to seek distant metastases with computed or positron emission tomography imaging.

Endocytoscopy is a novel means of diagnosing gastrointestinal lesions [9-14]. An endocytoscope has a single lens at its tip that can be configured to provide ultra-high magnification of conventional endoscopic images, achieving a magnification of approximately $\times 400$ over a $700 \mu \mathrm{m} \times$ $600 \mu \mathrm{m}$ area. The endocytoscopy system allows observation of living gastrointestinal cells in situ, including their nuclei and blood supply. This allows real-time assessments to be made of their histologic characteristics and thus can assist with the immediate diagnosis of gastrointestinal lesions. The combination of endocytoscopy and 

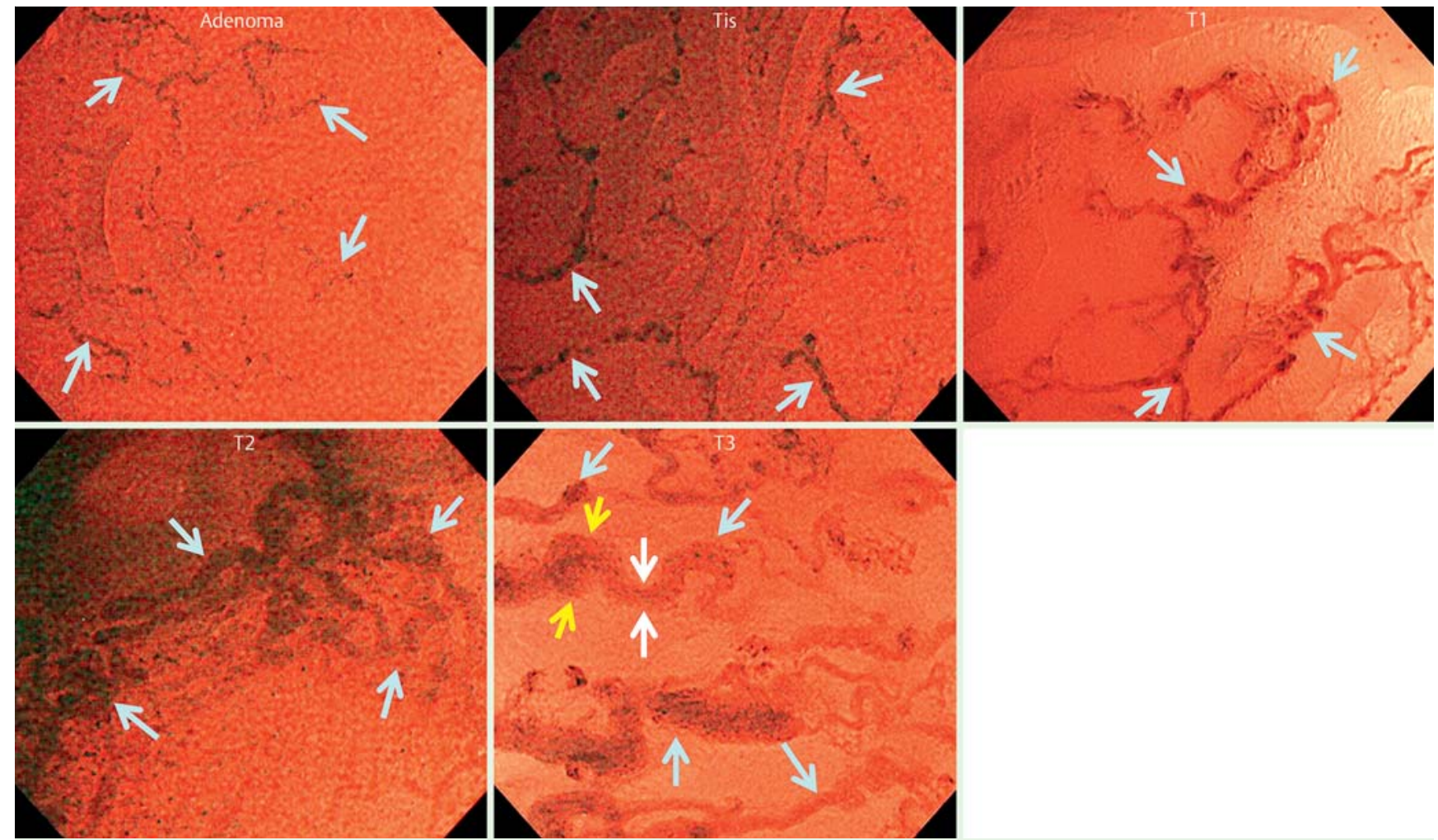

Fig. 1 Images obtained by endocytoscopy with narrow-band imaging (EC-NBI) at ultra-high magnification of approximately $\times 400$ for each depth of tumor invasion. Microvessels are visible as dark brown lines on EC-NBI images (blue arrows). In EC-NBI images, we defined the mean diameter of four vessels as the "tumor vessel diameter' and the proportional difference between the maximum and minimum vessel diameters as "tumor vessel caliber variation". The yellow arrows in the panel showing the T3 lesion indicate the maximum diameter and the white arrows the minimum diameter of a vessel.

NBI systems (EC-NBI) provides more detailed information about tumor vessels, including tumor blood flow and minute variations in the characteristics of tumor vessels [15].

Observing tumor vessel patterns makes it possible to predict vessel permeation [16]. In this retrospective pilot study, we examined the relationships between tumor vessel variation and venous or lymphatic vessel tumor permeation, by evaluating tumor vessel images obtained with an EC-NBI system.

\section{Materials and methods}

$\nabla$

We retrospectively studied lesions that had been treated by endoscopic or surgical resection after observation by EC-NBI at Showa University Northern Yokohama Hospital between February 2009 and September 2014. We analyzed only lesions that had been observed with EC-NBI by an endoscopist before treatment that were ultimately diagnosed as tubular adenomas or adenocarcinomas on subsequent histopathologic examination. Exclusion criteria included lesions that had been treated with chemotherapy or radiation therapy beforehand, histology other than tubular adenoma or adenocarcinoma (such as tubulovillous adenoma, mixed histology with a poorly differentiated component and hamartomatous polyp), and lesions for which the vascular images were unclear or inadequate for the assessment of tumor microvessels. Histologic type may influence vessel diameter or structure $[17,18]$. To reduce potential bias caused by histologic type, we focused on tubular lesions. Our institution's ethics committee approved conduct of this pilot study, and waived the requirement for consent because of its retrospective design.

\section{Colonoscopy and endoscopic diagnosis}

We obtained ultra-high magnification images with an integrated-type endocytoscope (CF-Y0020-I prototype; Olympus, Tokyo, Japan) using a video endoscopic system (Evis Lucera Spectrum or Evis Lucera Elite, Olympus). We used an EC-NBI system to obtain information about tumor vessels.

\section{Measurement of tumor vessels}

We imported all endocytoscopy images to a personal computer and measured their vessels using the appropriate software (http://www.forest.impress.co.jp/library/software/hakarundesu/ [in Japanese]). An endocytoscopy image covers a $700 \mu \mathrm{m} \times 600$ $\mu \mathrm{m}$ area producing an image of 512 pixels $\times 454$ pixels; therefore, the length of one pixel is equivalent to almost $1.3 \mu \mathrm{m}$. To reduce the potential for bias when measuring tumor vessels, we defined the mean diameter of four vessels as "tumor vessel diameter" and the proportional difference between the maximum and minimum vessel diameters as "tumor vessel caliber variation" $(\bullet$ Fig. 1). We selected representative vessels for measurement; for example, we excluded extremely wide vessels in marginal areas of a protruding tumor. For tumor vessel caliber variation, we selected representative vessels with the greatest proportional difference in their diameters. The researchers who measured the vessels were not aware of the histopathologic findings. We examined the relationships between tumor vessel diameter, tumor vessel caliber variation, and venous or lymphatic vessel permeation. As depth of tumor invasion is thought to influence tumor vessel diameter, we analyzed the relationship between tumor vessel diameter, tumor vessel caliber variation, and depth of tumor invasion as a sub-study. 


\section{Histopathologic evaluation}

All specimens obtained at endoscopy or during surgical resection were fixed in $10 \%$ formalin, embedded in paraffin wax, and sliced into 3-mm sections. These were stained with hematoxylin and eosin, or with monoclonal antibodies against CD34 (specific for venous vessels), D2 - 40 (specific for lymphatic vessels) and desmin (specific for the muscularis mucosa and used to judge the depth of tumor invasion). All sections were examined by pathologists who were unaware of the results of microvessel measurement. Tumor invasion depth was classified based on the seventh edition of the Union for International Cancer Control TNM classification [19].

\section{Statistical analysis and validation}

Continuous variables are expressed as mean \pm standard deviation (SD). We used Student's independent $t$ test and Fisher's exact test to compare continuous variables. To obtain intraclass correlation coefficients (ICC), we used R version 2.13.1 ( $\mathrm{R}$ Foundation for Statistical Computing, Vienna, Austria, http://www.r-project.org/); other analyses were performed using SPSS for Windows version 10 (SPSS, Chicago, Illinois, United States).

We calculated the ICCs for tumor vascular diameter for validation. Two investigators (KT and KK) independently selected four representative tumor vessels from a selection of EC-NBI images. Two weeks after initial assessment, the same images were randomly shown to the same investigators for re-assessment. We defined ICC values of $0.0-0.20$ as slight agreement, $0.21-0.40$ as fair agreement, $0.41-0.60$ as moderate agreement, $0.61-0.80$ as substantial agreement, and $0.81-1.00$ as almost perfect agreement.

\section{Results}

\section{$\nabla$}

In all, 268 lesions were observed by EC-NBI before treatment. Of these lesions, 52 were excluded on histopathologic criteria, one because of previous chemoradiotherapy and 27 as the vascular images were unclear due to local oozing. Ultimately, 188 colorectal lesions from 188 patients were studied, including 39 adenomas and 149 differentiated adenocarcinomas (64 tumors in situ (Tis), $56 \mathrm{~T} 1$ carcinomas, $14 \mathrm{~T} 2$ carcinomas, and $15 \mathrm{~T} 3$ carcinomas; - Fig.2). In all, 752 vessels that were clearly visible on EC-NBI images were evaluated ( $\bullet$ Fig. $\mathbf{1}$ ).

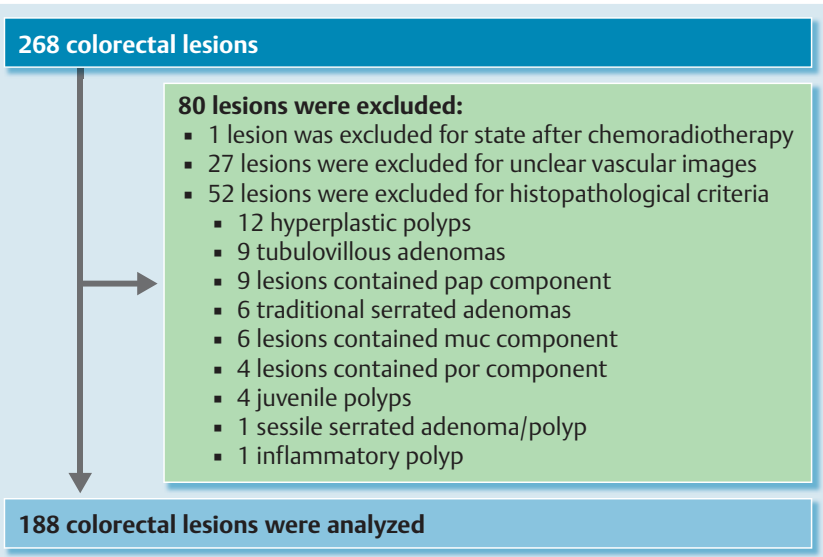

Fig. 2 Study flow chart.

The ICC $(1,2)$ of investigator KT was 0.988 (95\% confidence interval [CI] $0.970-0.995)$ and of investigator $\mathrm{KK}$ was $0.988(95 \% \mathrm{Cl}$ $0.970-0.995)$. The ICC $(2,2)$ between investigator KT and KK was 0.980 (95\%CI 0.949-0.992). Thus, they achieved almost perfect agreement.

There were significant differences in tumor vessel diameter and caliber variation between Tis lesions and $\mathrm{T} 1-\mathrm{T} 3$ carcinomas ( $\bullet$ Table1). Tumor vessel diameter and tumor vessel caliber variation increased with increasing depth of tumor invasion. There were significant differences in tumor vessel diameter and tumor vessel caliber variation between Tis lesions and T1 carcinomas, and between T1 and T2 carcinomas $(\mathrm{p}<0.01)(-$ Table2). Vessel permeation was not found in any Tis lesions. In T1 - T3 carcinomas, tumor vessel diameter and caliber variation were greater in tumors where there was venous or lymphatic vessel permeation compared with those in which there was no evidence of venous or lymphatic permeation ( $\triangle$ Table 3 ).

As depth of tumor invasion may have influenced our findings, its relationships with tumor vessel diameter and tumor vessel caliber variation were analyzed. In T1 carcinomas, tumor vessel diameter and tumor vessel caliber variation were significantly greater when there was venous vessel permeation ( $\bullet$ Table 4$)$. In T2 and T3 carcinomas, tumor vessel caliber variations were again significantly greater when there was venous permeation ( 0 Table 4). Tumor vessel caliber variation was significantly greater when there was lymphatic vessel permeation in $\mathrm{T} 3$ carcinomas ( $\odot$ Table4; $\odot$ Fig.3, $\odot$ Fig.4, $\odot$ Fig.5, $\odot$ Fig. 6).

\begin{tabular}{|lccl|}
\hline & Tis $(\mathbf{n}=\mathbf{6 4})$ & T1 $\mathbf{T} \mathbf{T}(\mathbf{n}=\mathbf{8 5})$ & $\boldsymbol{P}$ value \\
\hline Tumor vessel diameter $(\mu \mathrm{m})$ & $20.6 \pm 4.8$ & $35.7 \pm 7.9$ & $<0.01$ \\
\hline Tumor vessel caliber variation & $0.273 \pm 0.061$ & $0.372 \pm 0.088$ & $<0.01$ \\
\hline
\end{tabular}

Table 1 Differences in tumor vessel diameter and caliber variation between tumors in situ (Tis) and $\mathrm{T} 1-\mathrm{T} 3$ carcinomas

\begin{tabular}{|lll|}
\hline Tumor invasion depth & Tumor vessel diameter $(\boldsymbol{\mu m})$ & Tumor vessel caliber variation \\
\hline Adenoma $(n=39)$ & $19.5 \pm 4.2$ & $0.277 \pm 0.115$ \\
\hline Tis $(n=64)$ & $20.6 \pm 4.8$ & $0.273 \pm 0.061$ \\
\hline T1 $(n=56)$ & $33.0 \pm 7.2$ & $0.342 \pm 0.080$ \\
\hline T2 $(n=14)$ & $41.5 \pm 8.1$ & $0.415 \pm 0.076$ \\
\hline T3 $(n=15)$ & $40.2 \pm 5.6$ & $0.440 \pm 0.074$ \\
\hline
\end{tabular}

Table 2 Relationship between tumor vessel diameter and caliber variation by depth of tumor invasion. 
Table 3 Relationship between tumor vessel permeation and tumor diameter or caliber variation in T1 - T3 carcinomas.

\begin{tabular}{|c|c|c|c|c|c|c|}
\hline & $v-(n=47)$ & $v+(n=38)$ & $P$ value & $l y-(n=63)$ & $l y+(n=22)$ & $P$ value \\
\hline Tumor vessel diameter $(\mu \mathrm{m})$ & $31.4 \pm 5.8$ & $40.9 \pm 7.1$ & $<0.01$ & $34.0 \pm 7.0$ & $40.3 \pm 8.8$ & $<0.01$ \\
\hline Tumor vessel caliber variation & $0.307 \pm 0.048$ & $0.451 \pm 0.053$ & $<0.01$ & $0.359 \pm 0.080$ & $0.408 \pm 0.102$ & 0.02 \\
\hline
\end{tabular}

$\mathrm{v}$, venous vessel permeation; ly, lymphatic vessel permeation.

Table4 Relationship between tumor vessel permeation, tumor vessel diameter and caliber variation by depth of tumor invasion.

\begin{tabular}{|c|c|c|c|c|c|c|c|}
\hline \multicolumn{2}{|l|}{$\begin{array}{l}\text { Tumor invasion } \\
\text { depth }\end{array}$} & \multirow{2}{*}{$\begin{array}{l}v-(\mathbf{n}=39) \\
30.3 \pm 5.4\end{array}$} & \multirow{2}{*}{$\begin{array}{l}v+(n=17) \\
39.3 \pm 6.8\end{array}$} & \multirow{2}{*}{$\begin{array}{l}\text { Pvalue } \\
<0.01\end{array}$} & \multirow{2}{*}{$\begin{array}{l}l y-(n=47) \\
32.1 \pm 6.4\end{array}$} & \multirow{2}{*}{$\begin{array}{l}l y+(n=9) \\
37.5 \pm 9.4\end{array}$} & \multirow{2}{*}{$\begin{array}{l}P \text { value } \\
0.04\end{array}$} \\
\hline $\mathrm{T} 1$ & $\begin{array}{l}\text { Tumor vessel } \\
\text { diameter }(\mu \mathrm{m})\end{array}$ & & & & & & \\
\hline & $\begin{array}{l}\text { Tumor vessel } \\
\text { caliber variation }\end{array}$ & $0.301 \pm 0.050$ & $0.437 \pm 0.049$ & $<0.01$ & $0.340 \pm 0.074$ & $0.354 \pm 0.112$ & 0.62 \\
\hline & & $v-(n=4)$ & $v+(n=10)$ & $P$ value & $l y-(n=10)$ & $1 y+(n=4)$ & $P$ value \\
\hline \multirow[t]{3}{*}{$\mathrm{T} 2$} & $\begin{array}{l}\text { Tumor vessel } \\
\text { diameter }(\mu \mathrm{m})\end{array}$ & $37.4 \pm 4.8$ & $43.1 \pm 8.7$ & 0.25 & $39.9 \pm 6.1$ & $45.5 \pm 11.9$ & 0.25 \\
\hline & $\begin{array}{l}\text { Tumor vessel } \\
\text { caliber variation }\end{array}$ & $0.328 \pm 0.032$ & $0.450 \pm 0.057$ & $<0.01$ & $0.427 \pm 0.077$ & $0.385 \pm 0.075$ & 0.37 \\
\hline & & $v-(n=4)$ & $v+(n=11)$ & $P$ value & ly $-(n=6)$ & $l y+(n=9)$ & $P$ value \\
\hline \multirow[t]{2}{*}{ T3 } & $\begin{array}{l}\text { Tumor vessel } \\
\text { diameter }(\mu \mathrm{m})\end{array}$ & $36.5 \pm 3.6$ & $41.5 \pm 5.7$ & 0.13 & $39.3 \pm 4.7$ & $40.8 \pm 6.3$ & 0.64 \\
\hline & $\begin{array}{l}\text { Tumor vessel } \\
\text { caliber variation }\end{array}$ & $0.345 \pm 0.010$ & $0.475 \pm 0.053$ & $<0.01$ & $0.393 \pm 0.064$ & $0.471 \pm 0.067$ & 0.04 \\
\hline
\end{tabular}

$v$, venous vessel permeation; ly, lymphatic vessel permeation.

\section{Discussion}

We found no significant differences in tumor vessel diameter and caliber variation between adenomas and Tis. There were, however, significant differences in tumor vessel diameter and caliber variation between Tis and T1 carcinomas, and between $\mathrm{T} 1$ and $\mathrm{T} 2$ carcinomas. It has previously been reported that microvascular characteristics determined by NBI are associated with depth of submucosal invasion [20]; our findings support this conclusion. We found that tumor vessel diameter varies widely, and that caliber variation is greater in more deeply invasive tumors. This suggests that tumor vessel caliber variation is a useful means of predicting depth of tumor invasion.

Our findings also suggest that an additional assessment of vascular pattern could enhance the ability to predict tumor vessel permeation, especially in T1 carcinomas. Because venous or lymphatic vessel permeation mandates additional surgical resection [8, 21 ], in cases when vessel permeation is suspected, the lesion should be resected en bloc and evaluated carefully to establish whether tumor vessel permeation has occurred. Although further study is needed to confirm our findings, our results add to the evidence base that informs endoscopists' clinical practice.

Given that venous vessel permeation influences prognosis, establishing the risk of permeation and therefore of disease being more widespread would inform management decisions, particularly the extent to which lymph node dissection should be undertaken [22]. Where there is concern about lymph node metastasis, lymph node dissection should be performed when resecting the primary lesion to minimize the risk of recurrence.

Our findings indicate that EC-NBI is capable of assessing malignant potential, depth of tumor invasion, and vessel permeation in real time. The consequent improved assessment of risk of metastasis could help endoscopists and colorectal surgeons decide between endoscopic and surgical resection.
Our study had three limitations: it was a single center retrospective pilot study, it relied on prototype equipment, and there may have been selection bias in the vessels assessed.

We have recently reported the development of a computer-aided diagnostic system for endocytoscopy imaging (EC-CAD) capable of diagnosing colorectal lesions [23]. The system has the potential to evaluate the risk of vessel permeation or depth of tumor invasion in real time, although further evaluation is required. Using an automated diagnostic system will reduce the selection bias of tumor vessels and will enable the measurement of each visible tumor vessel immediately. Our findings indicate that the system has the potential to evaluate the risk of vessel permeation or depth of tumor invasion in real time.

Our findings also suggest that the characteristics of tumor microvessels can help predict prognosis, likely because angiogenesis is necessary for tumor growth $[24,25]$. The fragility of the tumor neovasculature and pressure on tissue caused by tumor growth may cause variations in vessel caliber. Venous permeation results in embolization of neovascular structures; the resultant intravascular pressure changes may also cause vessel caliber variation. It is difficult to draw any firm conclusions about the clinical relevance of these mechanisms, because of the difficulties of achieving precise correspondence between the endocytoscopy images and histopathologic findings. Nonetheless, an understanding of the characteristics of microvascular changes associated with vessel permeation will help inform endoscopists' treatment strategy for lesions predicted to be $\mathrm{T} 1$ carcinomas based on pit or vascular patterns. Presently, vessel permeation can only be diagnosed by histopathologic examination. The EC-NBI techniques could allow the endoscopist to resect a highly suspicious lesion en bloc during the same treatment episode, obviating the need for further endoscopy or surgery. Although further research is needed, we believe that EC-NBI holds great potential to improve the treatment and outcomes of patients with colorectal cancer. 


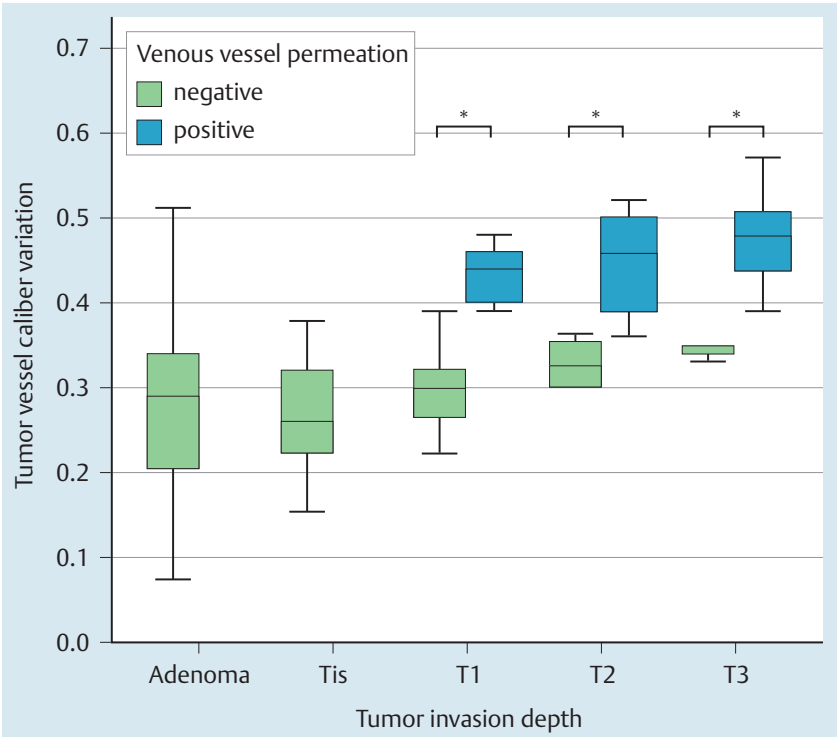

Fig. 3 The relationship between tumor vessel caliber variation and the presence or absence of venous vessel permeation by depth of tumor invasion. Only T1, T2, and T3 tumors showed evidence of venous permeation. Tumor vessel variation was significantly greater when there was venous permeation for all three categories of carcinoma $\left({ }^{*} P<0.01\right)$.

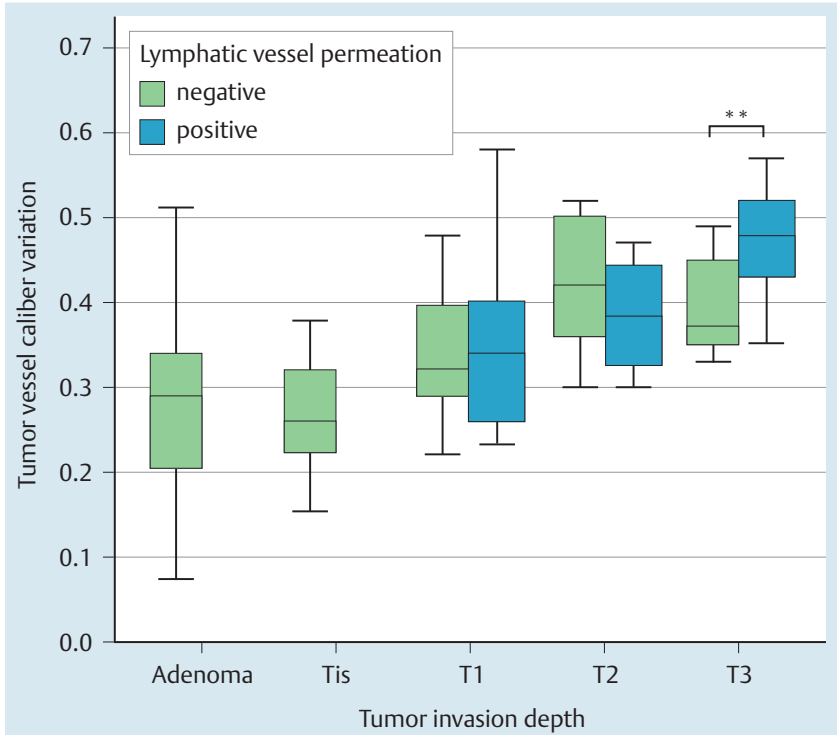

Fig. 4 The relationship between tumor vessel caliber variation and the presence or absence of lymphatic vessel permeation by depth of tumor invasion. Only T1, T2, and T3 lesions showed evidence of lymphatic vessel permeation. Tumor vessel variation was significantly greater in T3 carcinomas when there was lymphatic permeation $\left({ }^{* *} P<0.05\right)$.

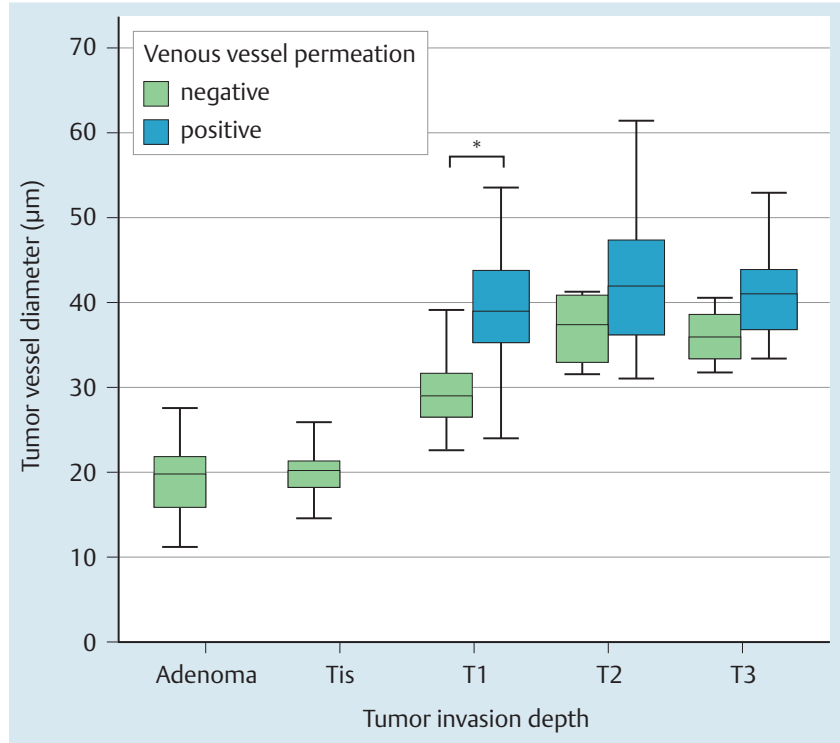

Fig. 5 The relationship between tumor vessel diameter and venous permeation by depth of tumor invasion. Only T1, T2, and T3 tumors showed evidence of venous permeation. Tumor vessel diameter was significantly greater in $\mathrm{T} 1$ carcinomas when there was venous permeation $\left({ }^{*} P<0.01\right)$.

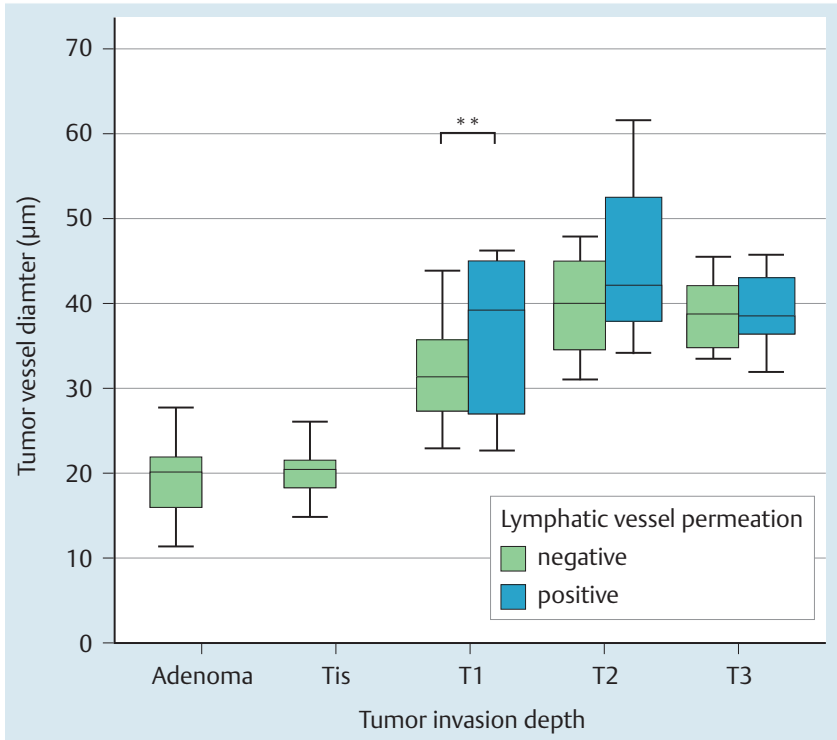

Fig. 6 The relationship between tumor vessel diameter and lymphatic vessel permeation by depth of tumor invasion. Only T1, T2, and T3 lesions showed evidence of lymphatic vessel permeation. Tumor vessel diameter was significantly greater in $\mathrm{T} 1$ carcinomas when there was lymphatic permeation $\left({ }^{* *} P<0.05\right)$. 
Competing interests: None

\section{Acknowledgments}

$\nabla$

Edanz Group Ltd (www.edanzediting.com) provided editorial assistance.

\section{References}

1 Kudo S, Hirota S, Nakajima T et al. Colorectal tumours and pit pattern. J Clin Pathol 1994; 47: 880-885

2 Kudo S, Tamura S, Nakajima T et al. Diagnosis of colorectal tumorous lesions by magnifying endoscopy. Gastrointest Endosc 1996; 44: 8-14

3 Sano Y, Horimatsu T, Fu KI et al. Magnifying observation of microvascular architecture of colorectal lesions using narrow-band imaging system. Dig Endosc 2006; 18: S44-S51

4 Wada Y, Kudo S, Kashida $\mathrm{H}$ et al. Diagnosis of colorectal lesions with the magnifying narrow-band imaging system. Gastrointest Endosc 2009; 70: $522-531$

5 Kanao H, Tanaka S, Oka S et al. Narrow-band imaging magnification predicts the histology and invasion depth of colorectal tumors. Gastrointest Endosc 2009; 69: 631-636

6 Ikematsu H, Matsuda T, Emura $F$ et al. Efficacy of capillary pattern type IIIA/IIIB by magnifying narrow band imaging for estimating depth of invasion of early colorectal neoplasms. BMC Gastroenterol 2010; 10: 33

7 Saito S, Tajiri H, Ohya $T$ et al. Imaging by magnifying endoscopy with NBI implicates the remnant capillary network as an indication for endoscopic resection in early colon cancer. Int J Surg Oncol 2011; 2011: 242608

8 Watanabe T, Itabashi M, Shimada Y et al. Japanese Society for Cancer of the Colon and Rectum (JSCCR) Guidelines 2014 for treatment of colorectal cancer. Int J Clin Oncol 2015; 20: 207-239

9 Kudo SE, Wakamura K, Ikehara $N$ et al. Diagnosis of colorectal lesions with a novel endocytoscopic classification - a pilot study. Endoscopy 2011; 43: 869-875

10 Kaise M, Ohkura Y, Iizuka T et al. Endocytoscopy is a promising modality with high diagnostic accuracy for gastric cancer. Endoscopy 2015; 47: $19-25$

11 Kutsukawa M, Kudo S, Ikehara $N$ et al. Efficiency of endocytoscopy in differentiating types of serrated polyps. Gastrointest Endosc 2014; 79: 648-656

12 Mori Y, Kudo S, Ikehara $N$ et al. Comprehensive diagnostic ability of endocytoscopy compared with biopsy for colorectal neoplasms: a pro- spective randomized noninferiority trial. Endoscopy 2013; 45: 98 105

13 Rotondano G, Bianco MA, Salerno R et al. Endocytoscopic classification of preneoplastic lesions in the colorectum. Int J Colorectal Dis 2010; 25: $1111-1116$

14 Cipolletta L, Bianco MA, Rotondano $G$ et al. Endocytoscopy can identify dysplasia in aberrant crypt foci of the colorectum: a prospective in vivo study. Endoscopy 2009; 41: 129-132

15 Kudo S, Misawa M, Wada $Y$ et al. Endocytoscopic microvasculature evaluation is a reliable new diagnostic method for colorectal lesions (with video). Gastrointest Endosc 2015; 82: 912 - 923

16 Weyn B, Tjalma WA, Vermeylen $P$ et al. Determination of tumour prognosis based on angiogenesis-related vascular patterns measured by fractal and syntactic structure analysis. Clin Oncol (R Coll Radiol) 2004; 16: $307-316$

17 Teixeira CR, Torresini RS, Canali $C$ et al. Endoscopic classification of the capillary-vessel pattern of colorectal lesions by spectral estimation technology and magnifying zoom imaging. Gastrointest Endosc 2009; 69: $750-756$

18 Kanemitsu T, Yao K, Nagahama T et al. The vessels within epithelial circle (VEC) pattern as visualized by magnifying endoscopy with narrowband imaging (ME-NBI) is a useful marker for the diagnosis of papillary adenocarcinoma: a case-controlled study. Gastric Cancer 2014; 17: 469-477

19 Sobin LH, Gospodarowicz MK, Wittekind C. TNM classification of malignant tumours. 7th edn. International Union Against Cancer. Chichester: Wiley-Blackwell; 2010

20 Hirata M, Tanaka S, Oka S et al. Evaluation of microvessels in colorectal tumors by narrow band imaging magnification. Gastrointest Endosc 2007; 66: $945-952$

21 Ueno H, Mochizuki H, Hashiguchi Yet al. Risk factors for an adverse outcome in early invasive colorectal carcinoma. Gastroenterology 2004; 127: $385-394$

22 Fujii T, Sutoh T, Morita $H$ et al. Vascular invasion, but not lymphatic invasion, of the primary tumor is a strong prognostic factor in patients with colorectal cancer. Anticancer Res 2014; 34: 3147-3152

23 Mori Y, Kudo SE, Wakamura K et al. Novel computer-aided diagnostic system for colorectal lesions by using endocytoscopy (with videos). Gastrointest Endosc 2015; 81: 621-629

24 Folkman J. Tumor angiogenesis: therapeutic implications. NEJM 1971; 285: $1182-1186$

25 Naumov GN, Akslen LA, Folkman J. Role of angiogenesis in human tumor dormancy: animal models of the angiogenic switch. Cell Cycle 2006; 5: $1779-1787$ 